\title{
Automating a Part of the Assessment of a University using Excel
}

\author{
Yoshitaka Kihara \\ Fukuyama University \\ Fukuyama, Hiroshima, Japan \\ kihara@fukuyama-u.ac.jp
}

\author{
Takashi Yamanoue \\ Fukuyama University \\ Fukuyama, Hiroshima, Japan \\ yamanoue@fukuyama-u.ac.jp
}

\author{
Motoo Tanaka \\ Fukuyama University \\ Fukuyama, Hiroshima, Japan \\ motanaka@fukuyama-u.ac.jp
}

\author{
Eiji Sato \\ Fukuyama University \\ Fukuyama, Hiroshima, Japan \\ sato@fukuyama-u.ac.jp
}

\author{
Yutaka Otsuka \\ Fukuyama University \\ Fukuyama, Hiroshima, Japan \\ yotsuka@fukuyama-u.ac.jp
}

\begin{abstract}
A process of establishing an assessment policy for a university and automating a part of the assessment is discussed. Excel was the tool used to carry out the automation. The form the assessment policy should take was discussed for three years, and was partially implemented in two months. We collected grades of all students of all classes from our university, using our academic affairs system. After the grade data were downloaded from the academic affairs system, we produced radar charts for each student, for each department, and for the university as a whole. Then, by clicking some buttons in the Excel book, the data were partitioned into the grade lists of all departments.
\end{abstract}

\section{CCS CONCEPTS}

- Social and professional topics $\rightarrow$ Professional topics; Professional topics.

\section{KEYWORDS}

Robotic Process Automation, Institutional Research, University Assessment, Excel

\section{ACM Reference Format:}

Yoshitaka Kihara, Takashi Yamanoue, Motoo Tanaka, Eiji Sato, and Yutaka Otsuka. 2019. Automating a Part of the Assessment of a University using Excel. In 2019 ACM SIGUCCS Annual Conference (SIGUCCS '19), November 3-6, 2019, New Orleans, LA, USA. ACM, New York, NY, USA, 6 pages. https: //doi.org/10.1145/3347709.3347821

\section{INTRODUCTION}

Most universities in the world are evaluated by an external body, such as WASC senior college and the university commission [4], to determine if applicable standards are met. The external body usually verifies that the subject university has satisfied the minimal standards for universities in the country or not, and it also verifies the Plan-Do-Check-Act (PDCA) cycle of the subject university is

Permission to make digital or hard copies of all or part of this work for personal or classroom use is granted without fee provided that copies are not made or distributed for profit or commercial advantage and that copies bear this notice and the full citation on the first page. Copyrights for components of this work owned by others than ACM must be honored. Abstracting with credit is permitted. To copy otherwise, or republish, to post on servers or to redistribute to lists, requires prior specific permission and/or a fee. Request permissions from permissions@acm.org.

SIGUCCS '19, November 3-6, 2019, New Orleans, LA, USA

(C) 2019 Association for Computing Machinery.

ACM ISBN 978-1-4503-5774-6/19/11 . .\$15.00

https://doi.org/10.1145/3347709.3347821 implemented and maintained correctly or not. Implementing the PDCA cycle requires an extensive amount of work and involves many people. Notably, the Check processes of the PDCA cycle requires extensive resources to collect evidence and to prove that the Plan was done correctly.

In Japan, the Ministry of Education, Culture, Sports, Science and Technology of Japan (MEXT Japan) issued the standards, "Quality Assurance Framework of Higher Education in Japan (2009)" [2]. It is said, "(2) A university shall, when assessing its students' academic achievement and approving their graduation, present them with a clear outline of the standards therefore, in advance, so as to ensure objectivity and rigidity, and shall conduct an assessment and approval process appropriately in accordance with said standards", in the "Article 25-2 (Publication of Standards for Evaluating Grades)" of the "Standards for Establishing Universities", in the framework of higher education. This assessment corresponding to the Check process of the PDCA cycle of the universities.

After the standards were shown, all universities in Japan were struggling to meet them.

Fukuyama University, a small university in the countryside of Japan, established a procedure for the assessment in spring of 2019. The university does not have enough staff to carry out the assessment by hand. Additionally, the university does not have the means to buy the assessment system, so the university decided to automate a part of the assessment using Excel. Using a kind of Robotic Process Automation (RPA) [5], the university succeeded in establishing an assessment procedure.

This paper outlines how the policy was made (section 2), what is the assesment policy (section 3), how the assessment was automated (section 4), the evaluation of the automated system (section 5), and an overview of related work (section 6).

\section{MAKING THE ASSESSMENT POLICY}

Fukuyama university was established in 1975. It is located in the rural area of Fukuyama city, Hiroshima Prefecture, Japan. The university has 3,884 students and 171 faculty members as of April 1st, 2019. The university issued the "Fukuyama University education system", which is the manifesto of reforming the education system of the university, in 2008 [1] and then revised it again in 2016. The university education center was established to realize the reformation in all educational aspects of the university, in 2009. Fukuyama University decided to establish the assessment policy, following the standards of MEXT [2], in line with other universities in Japan, and 
the university education center has been in charge of establishing the university assessment policy. Assessments, which are generated by the assessment policy, should be generated with minimal cost and minimal labor. So, ways of automating the generation of assessments at minimal costs were investigated from 2016. According to the standards set by MEXT, assessments should be "objectivity and rigidity". Therefore, we began to explore different options of assessments which are suitable for automated generation and which meet the standards of "objectivity and rigidity". In 2016, the university education center made the prototype policy, which was made to realize the automation, "objectivity" and "rigidity". The university education center showed the prototype policy to its committee members in the January 2017 committee meeting. In the committee meeting, every department was asked to make their own assessment policy proposal, by referring to the prototype policy, by June of 2017. All departments' assessment policy proposals were shown in the committee meeting of the university education center in June of 2017. Every department was asked to improve their assessment policy proposal by referring to other departments' proposals, by July of 2017. During August and September of 2017, automating the generation of assessments had been investigated, and the prototype of the automatic assessments generation system was made by a small number of faculty members. The automatic assessment generation system underwent a series of improvements leading up to the current system. All improved assessment policy proposals were shown in the committee meeting of the university education center in September of 2017. The status of making assessment policy and planned schedule to establish the assessment policy were shown in the committee meeting of the university education center in December of 2017. The prototype assessments which are generated automatically using grades of the fiscal year of 2017 were shown to the committee members of the university education center in the committee meeting of the center in September 2018. The policy was approved in the meeting. The policy also has been approved in the council meeting of the university in November 2018. The assessments of fiscal year 2018 have been generated in April of 2019.

\section{THE ASSESSMENT POLICY OF THE UNIVERSITY}

The assessment policy of Fukuyama university consists of procedures of assessments at the student level, the department level, and the university level.

The following subsections show each level of the assessment.

\subsection{Student Level Assessment}

The assessment of a student is generated by the following. a. The grade of a subject: The grade of a subject is determined in accordance with the assessment breakdown as is laid out in the syllabus of each course and shows the achievement of the following criterion. The achievement of the object of the subject is confirmed by the grade.

4.0: Excellent (Exceptional Achievement) in the subject. 3.0: Good (Extensive Achievement) in the subject. 2.0: Satisfactory (Acceptable Achievement) in the subject. 1.0: Poor (Minimal Achievement) in the subject.

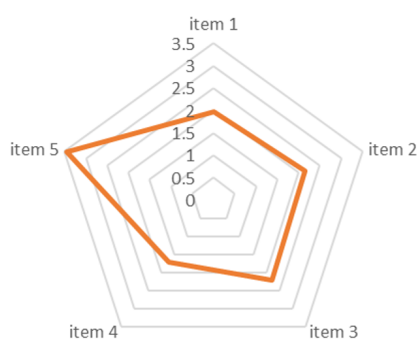

Figure 1: Radar chart which shows the gained quality of intermediate items.

b. The grade of the quality: Diploma policy for each department is a combination of achieved qualities of intermediate items. An intermediate item is a more global skill such as "acquire culture as a member of society", "acquire basic scientific ability", or so on. The grade of a subject of a student indicates some level of the qualities and it is shown in the syllabus. The gained quality of the intermediate item $\mathrm{i}$ of the student is calculated by the equation (1).

$$
G Q_{i}=\frac{\sum_{j} G_{j} \times U_{j} \times W_{i j}}{\sum_{j} U_{j} \times W_{i j}}
$$

In the equation (1), $G Q_{i}$ shows the gained quality of the intermediate item $\mathrm{i}$ of the student. $G_{j}$ shows the grade of the $j$-th subject of the student. $U_{j}$ shows the unit number of the $j$-th subject. $W_{i j}$ shows the $i$-th weight for the $j$-th subject. The followings show the meaning of the value of the weight $\left(W_{i j}\right)$.

3.0: The subject $\mathrm{j}$ matches the intermediate item i completely

2.0: The subject $\mathrm{j}$ matches the intermediate item $\mathrm{i}$ approximately

1.0: The subject $\mathrm{j}$ matches the intermediate item i partly

Table 1 shows an example which of how the gained quality is calculated. Figure 1 shows the radar chart which corresponds to the gained quality of intermediate items. This chart is generated from the Table 1 and shows the assessment of the student.

\subsection{Departments' Level Assessment}

Assessments of a department are degrees of achievement which are generated by calculating the proportion of graduated students who have achieved the designated grade of the quality of intermediate items of the department. The designated grade of the quality of intermediate items of the department are defined in the table of the assessment policy of the department. The degree 4, the maximum degree, means that $75 \%$ or more of the students have achieved the designated grade of the intermediate item. The degree 3 means that $50 \%$ to $75 \%$ of the students achieved the designated grade of the intermediate item. The degree 2 means that $25 \%$ to $50 \%$ of the students achieved the designated grade of the intermediate item. The degree 1 means that $25 \%$ or less of the students achieved the designated grade of the intermediate item. 
Table 1: Example of How the gained quality is calculated

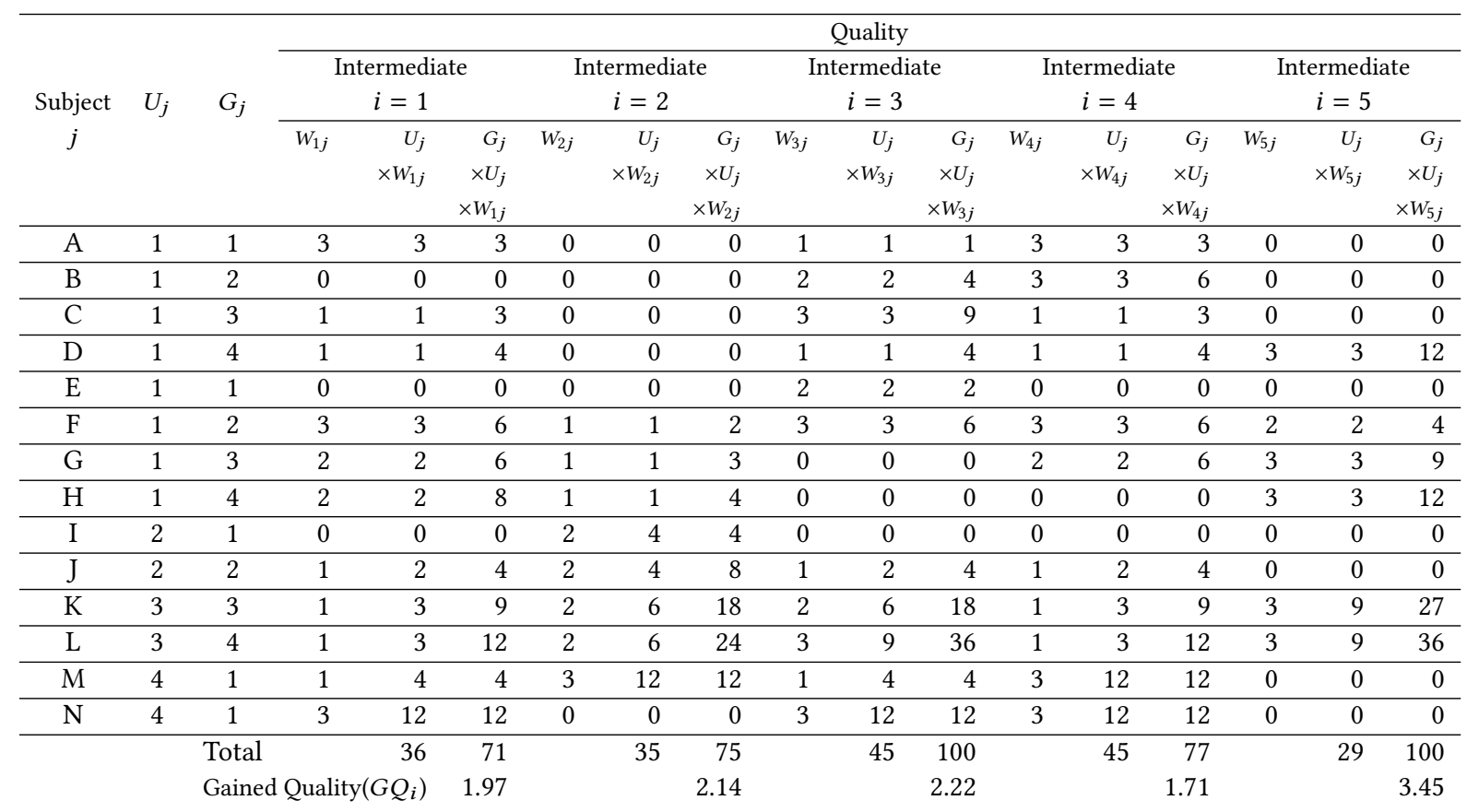

Table 2: A part of simulation of proportion of graduated students who achieved the designated grade.

\begin{tabular}{|c|c|c|c|c|c|c|c|c|}
\hline & & $\begin{array}{l}\text { Proport } \\
\text { the } G Q\end{array}$ & $\begin{array}{l}\text { on of st } \\
\text { of the ir }\end{array}$ & $\begin{array}{l}\text { lents wh } \\
\text { ermedia }\end{array}$ & $\begin{array}{l}\text { achieve } \\
\text { item }\end{array}$ & & & \\
\hline & $G Q$ & item 1 & item 2 & item 3 & item 4 & item 5 & item 6 & $\ldots$ \\
\hline \multirow{4}{*}{ Department 1} & $3.3-$ & $4 \%$ & $9 \%$ & $10 \%$ & $8 \%$ & & & \\
\hline & $2.8-3.3$ & $13 \%$ & $20 \%$ & $26 \%$ & $24 \%$ & & & \\
\hline & $2.0-2.8$ & $57 \%$ & $64 \%$ & $61 \%$ & $62 \%$ & & & \\
\hline & $1.0-2.0$ & $26 \%$ & $6 \%$ & $3 \%$ & $5 \%$ & & & \\
\hline \multirow{4}{*}{ Department 2} & $3.3-$ & $14 \%$ & $22 \%$ & $17 \%$ & $28 \%$ & $17 \%$ & $17 \%$ & \\
\hline & $2.8-3.3$ & $33 \%$ & $44 \%$ & $36 \%$ & $42 \%$ & $42 \%$ & $39 \%$ & \\
\hline & $2.0-2.8$ & $47 \%$ & $31 \%$ & $42 \%$ & $25 \%$ & $39 \%$ & $36 \%$ & \\
\hline & $1.0-2.0$ & $6 \%$ & $3 \%$ & $6 \%$ & $6 \%$ & $3 \%$ & $3 \%$ & \\
\hline
\end{tabular}

In order to decide the designated grade, we have simulated the proportion of graduated students, who achieved the designated grade, by varying the designated grade.

Table 2 shows a part of the simulation. We have decided to use 2.5 for the designated grade. Table 3 shows a part of assessments of department which shows degrees of achievement of each department.

\subsection{The University Level Assessment}

The assessment of the university is degrees of achievements which are generated by calculating the proportion of graduated students who achieved the designated grade of the quality of competencies of the university. Competencies are "broader culture (C-1)", "high level knowledge of specialties (C-2)", "ability of application (C3)", "ability of practice (C-4)", "motivation and endurability (C-5)", "cooperativeness with others (C-6)" and "responsibility and ethics (C-7)". The grades of the competencies are calculated as they were in 2.1 using, the weights for subjects and competencies. The designated grades of the quality of competencies of the university are defined in the table of the assessment policy of the university. The degree 4, the maximum degree, means that $75 \%$ or more of students achieved the designated grade of the elemental item. The degree 3 means that $50 \%$ to $75 \%$ of students achieved the designated grade of the elemental item. The degree 2 means that $25 \%$ to $50 \%$ of students achieved the designated grade of the elemental item. The degree 1 means that $25 \%$ or less of students achieved the designated grade of the intermediate item. 
Table 3: A part of degree of achievements of departments

\begin{tabular}{|c|c|c|c|c|c|c|c|}
\hline & \multicolumn{5}{|c|}{ Degree of Achievements of the Department } & & \\
\hline Department & item 1 & item 2 & item 3 & item 4 & item 5 & item 6 & $\ldots$ \\
\hline Department 1 & 2 & 3 & 3 & 3 & 3 & & \\
\hline Department 2 & 3 & 4 & 4 & 4 & 4 & 4 & \\
\hline
\end{tabular}

Table 4: The provisional assessment of the university

\begin{tabular}{|c|c|c|c|c|c|c|c|}
\hline & $\mathrm{C}-1$ & $\mathrm{C}-2$ & $\mathrm{C}-3$ & $\mathrm{C}-4$ & C-5 & C-6 & $\mathrm{C}-7$ \\
\hline University's & & & & & & & \\
\hline assessments & 3 & 3 & 4 & 4 & 3 & 4 & 4 \\
\hline & \multicolumn{7}{|c|}{ C-1: Broader culture } \\
\hline & \multicolumn{7}{|c|}{ C-2: High level knowledge of specialities } \\
\hline & \multicolumn{7}{|c|}{ C-3: Ability of applicaiton } \\
\hline & \multicolumn{7}{|c|}{ C-4: Ability of practice } \\
\hline & \multicolumn{7}{|c|}{ C-5: Motivation and endurability } \\
\hline & \multicolumn{7}{|c|}{ C-6: Cooperativeness with others } \\
\hline & \multicolumn{7}{|c|}{ C-7: Responsibility and ethics } \\
\hline
\end{tabular}

Table 4 shows the provisional assessment of the university which is generated by the procedures of 2.1,2.2. and 2.3.

\section{AUTOMATING A PART OF THE ASSESSMENT}

Generating assessments for only one student is not so difficult. It can be performed using just one Excel sheet with several operations. However, we have to generate assessments for all students of all departments, assessments for all departments, and the assessment for the university. It is almost impossible to generate them by hand with a limited number of staff. Fortunately, we are using an academic affairs system in which all of the grades of all of the subjects of all of the students are stored in the system in machine readable format. Generating assessments has to be performed twice a year, when every grade of all subjects of all students acquired in the academic affairs system at end of semesters. It does not need to be performed every time a student wants to see the assessment. Unfortunately, extending the affairs system, to be able to generate assessments, is not easy. It requires negotiation between us and the vendor of the system, it requires precise specification of the generation, and it is not easy to change the specification after the purchase of the extending. So, we have tried to automate the generation by ourselves using Excel and have developed the assessment generator for students, departments, and the university as a whole.

The assessment generator for students inputs the grade list of all subjects of all students of a department (Grade-List), and outputs the assessment report (Reports) for each student of the department. The radar chart is included in the report.

The assessment generator for students is an Excel book. The Excel book of the assessment generator includes the following sheets.

a. Document of how to use the assessment generator

b. List of students of one department (Student-List) c. Working sheet for keeping the list of grades of a student (Working-1)

d. Weight table (Weight-Table). Weights, which show relationships between the grade of a subject and an intermediate item of the quality, are described in this table. This table is corresponding to the department's assessment policy. For example, the number of rows was 132 and the number of columns was 29 in the case of the department of pharmacy.

e. Notation-Value convert table (NV-Table). This table just describes the relationship between notation for weights and its values.

f. Working sheet for calculating $G Q$ values (Working-2). This sheet is corresponding to the Table 1 in the section 2.1. GQs of all subjects of a student are calculated and assigned in cells of this sheet by the values and expressions in this sheet. When the Working- 1 sheet is filled with the grades data of a student, GQs of the student are calculated and assigned automatically using Excel's vlookup functions. In the case of the department of pharmacy, the number of rows was 147 and the number of columns was 114 .

g. Framework of the assessment report of a student (AssessmentFramework). The assessment report of a student is generated in the format of this framework. This sheet just re-format the Working-2 sheet.

The Excel book of the assessment generator also includes the following Macros

a. Student List Reader (List-Reader). This macro has 19 lines.

b. Assessment Report Maker (Report-Maker). This macro has 32 lines.

Assessments for all students of a class are generated by the following steps using the Excel book and a Windows based personal computer. (We are currently doing the calculations on a Mac Book Pro).

Step 1. Download the grade list of all students of all departments (all list) from our academic affairs system.

Step 2. Partition the 'all list' into grade lists for each department (department list). This is performed by hand because it is easy and it is not time consuming work; however, it would also be easy to automate.

Step 3. For each department, prepare the Excel book for the department and perform the following sub steps.

Step 3.1 Place the department list in the designated folder. Step 3.2 Write the year and semester of the assessment in the designated cells in the Working-2 sheet.

Step 3.3 Click the button in the Student-List sheet of the Excel Book. The student list of the department is generated in the Student-List sheet by this action. 
Table 5: Estimated effort for making and running the assessment generators for students.

\begin{tabular}{|c|c|c|c|c|c|c|}
\hline \multirow[t]{2}{*}{$\begin{array}{l}\text { Estimated Effort } \\
(\text { man } \times \text { hour })\end{array}$} & \multicolumn{2}{|c|}{$\begin{array}{l}\text { Excel without } \\
\text { vlookup nor } \\
\text { Macro }\end{array}$} & \multicolumn{2}{|c|}{$\begin{array}{l}\text { Excel with } \\
\text { vlookup and } \\
\text { without Macro }\end{array}$} & \multicolumn{2}{|c|}{$\begin{array}{l}\text { Excel with } \\
\text { vlookup and } \\
\text { Macro }\end{array}$} \\
\hline & $\operatorname{man}$ & machine & $\operatorname{man}$ & machine & man & machine \\
\hline Making Weight-Table & 5 & 0 & 5 & 0 & 5 & 0 \\
\hline $\begin{array}{l}\text { Making Weight-Table } \\
\text { for all departments }\end{array}$ & 20 & 0 & 20 & 0 & 20 & 0 \\
\hline $\begin{array}{l}\text { Making Table } 1 \\
\text { (Working-2) }\end{array}$ & 3 & 0 & 6 & 0 & 6 & 0 \\
\hline $\begin{array}{l}\text { Making Table } 1 \\
\text { for all departments }\end{array}$ & 10 & 0 & 25 & 0 & 25 & 0 \\
\hline Assessment-Framework & 3 & 0 & 5 & 0 & 5 & 0 \\
\hline $\begin{array}{l}\text { Assessment-Framework } \\
\text { for all departments }\end{array}$ & 10 & 0 & 20 & 0 & 20 & 0 \\
\hline List-Reader & 0 & 0 & 0 & 0 & 10 & 0 \\
\hline Report-Maker & 0 & 0 & 0 & 0 & 40 & 0 \\
\hline Generate One Student & 1 & 0 & 0.2 & 0 & 0.1 & 0.01 \\
\hline Generate 100 Students & 100 & 0 & 20 & 0 & 0.1 & 0.3 \\
\hline Generate 4000 Students & 4000 & 0 & 800 & 0 & 1 & 12 \\
\hline $\begin{array}{l}\text { Total(all departments } \\
\text { and } 4000 \text { students) }\end{array}$ & 4040 & 0 & 865 & 0 & 116 & 12 \\
\hline
\end{tabular}

Step 3.4 Click the button in the Working-2. All assessments for all students of the department of the semester and the year is generated by this action.

The List-Reader macro is executed in step 3.3. This macro makes the student list of the department from the grade list of the department.

The Report-Maker macro is executed in the step 3.4. This macro makes the grade list for each student in the student-list, copies the grade list into the Working-1 sheet, and generates the assessment report by copying the result in the Assessment-Framework sheet into the corresponding files.

An assessment generator for a department is similar to the assessment generator for students, except the Excel book has the function to calculate the proportion of students' achievement of designated GQs in the department. The Excel book also has another weight table for calculating the university's assessment. The assessment generator for a department generates the assessment for the department and data for calculating the university level assessment.

The assessment generator for the university inputs the data, which are generated by assessment generators for all departments, and generates the university level assessment.

\section{EVALUATION OF THE AUTOMATING}

We would like to know how effective the automating the assessment generation is. It took about two months of work carrying out in faculty members' spare time to make the automatic assessment generator for students as noted in section two. It is not questionable to use Excel in such case today. So, we have compared the estimated effort with or without using vlookup function and with or without using macros. As shown in section 4, vlookup functions make the calculation of Working-2 automatic. If we could not use vlookup functions, calculating the Working-2 sheet became cumbersome work.

Table 5 shows the results of comparation of the estimated effort. Using vlookup functions was much more effective than not using vlookup functions. Using vlookup functions and macros was the most effective. As shown in the section four, the macros of the automatic assessment generator are very short. So, the estimations of Making List-Reader and Making Report-Maker are pessimistic.

\section{RELATED WORK}

\subsection{WASC Senior College and University Commission}

The WASC Senior College and University Commission ("the Commission") [4] is a regional accrediting agency serving a diverse membership of public and private higher education institutions throughout California, Hawaii, and the Pacific as well as a limited number of institutions outside the U.S. The commission made the "Rubric for Assessing Educational Effectiveness". Our process of establishment of assessment policy is influenced by this rubric as are the assessments of some other Japanese universities.

\subsection{Visualization of Achievements in Japanese Universities}

MEXT Japan is carrying out the Acceleration Program for University Education Rebuilding (AP). This program support universities which promote the effort of rebuilding, along with the suggestion of education recover meeting of Japanese government. The AP includes the theme of "visualization of achievement"[3]. There were eight universities and technical colleges, whose applications were accepted. Two of these institutions also use radar charts for visualizing the achievement of students. However, according to 
the brochures of the eight universities, none has established an assessment policy for their organization.

\subsection{YU CoB CuS}

YU CoB CuS, Yamaguchi University Competency-Based Curricular System [6], shows how the student acquires the items in the diploma policy quantitatively by a radar chart for each student when they graduate. The way of making the radar chart is almost the same as ours. However, the YU CoB CuS does not mention the assessment policy of their organization.

\subsection{Robotic Process Automation}

Robotic Process Automation (RPA) [5] is an application of technology, governed by business logic and structured inputs, aimed at automating business processes. There are many RPA tools in the world and they are very similar to Excel macros. Some articles on the web say RPA is not the same as an Excel macro because a knowledge of programming is required to use Excel macro. However, Excel macro has a long history and there are many people who are comfortable using it. It is difficult to predict which RPA tool survives except Excel macro, because there are many Excel macros and many know how to use Excel on the web.

\section{CONCLUSION}

We have established the assessment policy of a university and we have developed automatic assessment generators for the assessment policy. Excel was used to realize the automation. We had to improve the assessment policy, and we had to operate and will have to maintain the assessment generators every year from now on.

Operation and maintaining of the assessment generation should not be performed by faculty members. However, every member, whether s/he is a faculty or not, is busy in our university. Who should perform the operation and maintenance of the system from now on is an open question. Outsourcing may be one of the best answers for this problem. However, outsourcing requires precise definition of the operation and maintenance. It is not flexible. So, someone in our university has to do this and this requires a shift in responsibilities from other duties. In order to reduce the disruption of current roles, all should be more comfortable using information and communication technology such as RPA.

\section{ACKNOWLEDGMENTS}

Establishing the assessment policy could not be realized without co-operation of all faculty members of our university. Automating generation of assessments could not be realized by co-operation of administrative staffs. We thank all of them.

\section{REFERENCES}

[1] Fukuyama University 2016. Fukuyama University Education System (revised version Fukuyama University.

[2] Ministry of Education, Culture, Sports, Science and Technology of Japan 2009. Quality Assurance Framework of Higher Education in Japan. Ministry of Education, Culture, Sports, Science and Technology of Japan. http://www.mext.go.jp/ component/english/__icsFiles/afieldfile/2011/06/20/1307397_1.pdf.

[3] Ministry of Education, Culture, Sports, Science and Technology of Japan 2019 Visualization of Outcome of Learning, Acceleration program for renovation of university education. Ministry of Education, Culture, Sports, Science and Technology of Japan.
[4] WASC. [n.d.]. WASC Senior College and University Commission. https://www. wscuc.org.

[5] Wikipedia [n.d.]. Robotic process automation. Wikipedia. https://en.wikipedia. org/wiki/Roboticprocessautomation.

[6] Yamaguchi University, Faculty of Global and Science Studies 2015. YU Cob Cus, Yamaguchi University Competency-Based Curricular System. Yamaguchi University, Faculty of Global and Science Studies. http://gss.yamaguchi-u.ac.jp/yu_cobcus/. 